\title{
Revenu des médecins et TARMED: ce n'est pas si simple!
}

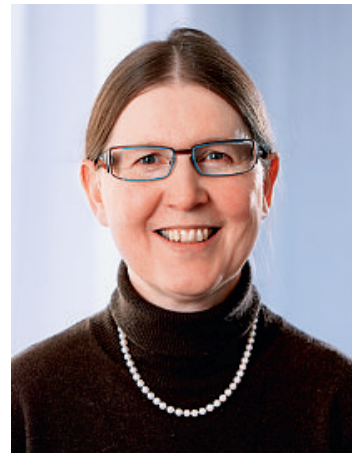

Marie-Christine Peter
Le $1^{\text {er }}$ avril 2009, journée de la médecine de famille, les médias et certains politiciens (dont les conseillers d'Etat Carlo Conti et Thomas Burgener) ont appelé à une montée aux barricades des médecins de premier recours contre les spécialistes afin d'améliorer les revenus des premiers nommés. La solution proposée peut sembler très simple à première vue: résilier les conventions TARMED afin de négocier de meilleures conditions pour les médecins de famille. Mais qu'est-ce que le TARMED et quelles possibilités offre-t-il?

\section{Si l'on réduit l'indemnisation des prestations}

\section{hospitalières, pas un centime n'ira au médecin de premier recours qui exerce une activité ambulatoire}

Le TARMED est un tarif calculé à l'origine selon des critères d'économie d'entreprise. Chaque prestation comporte une partie médicale (qui couvre le salaire du médecin) et une partie technique (incluant le loyer, les investissements, les salaires, les assurances, etc). Certaines prestations qui exigent des compétences particulières sont mieux indemnisées par le biais d'une «valeur intrinsèque»; elles sont fournies principalement dans le domaine hospitalier (stationnaire et ambulatoire). Si l'on réduit l'indemnisation de ces prestations hospitalières, pas un centime n'ira au médecin de premier recours qui exerce une activité ambulatoire. La FMH a analysé les effets du TARMED sur la composante médicale des prestations ambulatoires fournies par les différentes sociétés de discipline et constaté que l'écart entre la société la moins et la plus chère était d'environ $20 \%$ seulement. En revanche, la composante technique pose problème car les paramètres instaurés à la fin des années 90 n'ont plus été adaptés depuis lors. Il en résulte que les coûts, notamment pour les disciplines relevant de la médecine de premier recours, ne sont plus couverts qu'à $60 \%$ par la composante technique. La FMH élabore actuellement un nouveau modèle de calcul pour les cabinets médicaux, plus transparent et mieux apte à refléter la réalité des coûts. Ce modèle devra tout d'abord être accepté par TARMED Suisse avant d'être approuvé par le Conseil fédéral.

Les revenus élevés de certains spécialistes ne proviennent que pour une part peu importante de l'assurance de base. Ils sont essentiellement générés par la fourniture de prestations relevant des assurances complémentaires de patients assurés en division privée ou semi-privée, ou alors de prestations non prises en charge par l'assurance de base. Les lois du libre marché sont ici applicables. En médecine de premier recours, il n'existe par contre que très peu de prestations non obligatoires que le médecin peut facturer librement: la médecine complémentaire en est cependant un exemple. A noter toutefois que malgré les prix liés au TARMED, la grande majorité des médecins qui pratiquent la médecine complémentaire souhaite son intégration dans le catalogue des prestations à prise en charge obligatoire, afin que tous les patients puissent en bénéficier - et cela même si $70 \%$ de la population dispose d'une assurance-complémentaire.

Une revalorisation substantielle de la médecine de premier recours dans le TARMED n'est pas possible, à moins d'en faire un «tarif de bazar» avec de nombreuses inégalités. Il n'est par exemple pas acceptable qu'à prestations égales, celles-ci soient plus chères chez le généraliste que chez le spécialiste. En revanche, certaines prestations fournies par le médecin de premier recours, telles que le service de garde, les visites à domicile ou les consultations urgentes pourraient être mieux rétribuées par une hausse des forfaits existants ou par la création de nouveaux forfaits. Les coûts supplémentaires ainsi engendrés seraient largement compensés par les économies réalisées dans les services d'urgences et, en partie

\section{Les prestations fournies par le médecin de premier recours, telles que le service de garde, les visites à domicile ou les consultations urgentes, devraient être mieux rétribuées par une hausse des forfaits existants ou par la création de nouveaux forfaits}

aussi, par un nombre réduit d'hospitalisations. Cela suppose toutefois que les médecins de premier recours soient à nouveau davantage disposés à fournir ce type de prestations, et aussi que les patients puissent recommencer à consulter leur médecin en dehors des heures de bureau. A cet effet, il faut développer, pour les cabinets médicaux, de nouveaux modèles créatifs assortis de conditions de travail respectueuses de la famille.

Dr Marie-Christine Peter, membre du Comité central de la FMH, Domaine Tarifs et conventions 